\title{
A Density Dependent Dispersion Correction
}

\author{
Stephan N. Steinmann§ and Clémence Corminboeuf*
}

§SCS Metrohm Prize for best oral presentation

\begin{abstract}
Density functional approximations fail to provide an accurate treatment of weak interactions. More recent, but not readily available functionals can lead to significant improvements. A simple alternative to correct for the missing weak interactions is to add, a posteriori, an atom pair-wise dispersion correction. We here present a density dependent dispersion correction, dDXDM, which dramatically improves the performance of popular functionals (e.g. PBE-dDXDM or B3LYP-dDXDM) for a set of 145 systems featuring both inter- and intramolecular interactions. Whereas the highly parameterized M06-2X functional, the long-range corrected LC-BLYP and the fully non-local van der Waals density functional rPW86-VV09 also lead to improved results as compared to standard DFT methods, the enhanced performance of dDXDM remains the most impressive.
\end{abstract}

Keywords: Density functional theory $\cdot$ Dispersion correction · Molecular interactions

\section{Introduction}

Kohn-Sham density functional theory[1] (DFT) provides a powerful framework for electronic structure computations and has become the preferred methodology for modeling many-body systems at reasonable computational cost. Despite their indisputable success, popular local and semilocal density functionals fail to describe long-range dispersion interactions, which typically dominate weakly bound intermolecular complexes. ${ }^{[2-5]}$ In addition, most popular functionals are unable to accurately treat seemingly simple hydrocarbon reaction energies. ${ }^{[6-9]}$ For instance, isomerization reactions and Pople's isodesmic bond separation equations (BSEs) ${ }^{[10-12]}$ of alkanes are very challenging. ${ }^{[6-8]}$ These DFT weaknesses are well illustrated by the dramatic underestimation ${ }^{[7]}$ of the propane bond separation Eqn. (1), a failure that originates mainly from the neglect of non-bonded interactions (see refs [13-15]

${ }^{*}$ Correspondence: Prof. Dr. C. Corminboeuf Laboratory for Computational Molecular Design Institut des Sciences et Ingénierie Chimiques Ecole Polytechnique Fédérale de Lausanne $\mathrm{CH}-1015$ Lausanne

E-mail: clemence.corminboeuf@epfl.ch for detailed discussion). Within this context, we have demonstrated that applying a dispersion correction tailored for weak intramolecular interactions can eliminate these errors. ${ }^{[16-19]}$

$$
\mathrm{C}_{3} \mathrm{H}_{8}+\mathrm{CH}_{4} \rightarrow 2 \mathrm{C}_{2} \mathrm{H}_{6}
$$

The approach followed by others ${ }^{[14,20-23]}$ and $\mathrm{us}^{[16-18]}$ is to improve the poor performance of traditional density functionals, a posteriori, by means of an atom pairwise dispersion correction (Eqn. (2)). [20-22] Dispersion-correcting potentials ${ }^{[24-28]}$ can be used for the same purpose but are not discussed here.

$$
E_{d i s p}=-\sum_{i=2}^{N_{a t}} \sum_{j=1}^{i-1} f\left(R_{i j} ; i ; j\right) \frac{C_{6}^{i j}}{R_{i j}^{6}}
$$

Eqn. (2) generally employs a crude estimate ${ }^{[22,29]}$ for the $\mathrm{C}_{6}$ dispersion coefficients. The damping function typically depends on fixed van der Waals radii and a few empirical parameters. In order to go beyond system-independent dispersion coefficients, Becke and Johnson (BJ) proposed the exchange-hole dipole moment (XDM) formalism. ${ }^{[30-32]}$ The XDM formalism, used in our scheme, relies on molecular densities and free atomic polarizabilities and generates (higher order) dispersion coefficients (i.e. $\mathrm{C}_{6}, \mathrm{C}_{8}$ and $\mathrm{C}_{10}$ ). Related schemes were developed by Tkatchenko and Scheffler ${ }^{[33]}$ and more recently by Sato and Nakai. ${ }^{[34,35]}$

Over the past decades, the shortcomings of conventional semi-local and hybrid density functionals have also motivated the development of more sophisticated and/or more accurate DFT functionals referred herein to as 'modern'. Their construction follows different approaches that aim to i) improve the treatment of dispersive interactions (i.e. by fitting semi-local and hybrid functionals to experimental and high-level $a b$ initio data, ${ }^{[36,37]}$ or by deriving fully non-local van der Waals density functionals $\left.{ }^{[38-41]}\right)$ and ii) reduce the 'delocalization error' ${ }^{[42-45]}$ (e.g. by supplementing the approximate short-range DFT exchange by exact exchange in the long-range ${ }^{[46-49]}$ ).

Although very instructive, the benchmarking of these latest approximations remains scarce as the methods are often implemented in developmental versions of codes and thus not readily available to the users.

We here present a density dependent dispersion correction ${ }^{[18]}$ and discuss the benefit of using the proposed scheme in conjunction with standard density functionals. The performance of the approach for treating inter- and intra-molecular weak interactions is then compared to that of 'modern' functionals.

\section{Methods and Computational Details}

Our correction, called dDXDM, ${ }^{[18]}$ uses $\mathrm{C}_{6}, \mathrm{C}_{8}$ and $\mathrm{C}_{10}$ dispersion coefficients computed according to BJ's XDM formalism. ${ }^{[30-32]}$ The coefficients are distributed among the atoms based on iterative ${ }^{[50]}$ (instead of classical[51]) Hirshfeld weights that better account for polarization effects. Akin to our former empirical correction, ${ }^{[17]}$ our procedure uses a damped Tang and Toennies damping function ${ }^{[52]}$ that eliminates the correction at covalent distances. The proposed damping function depends on the density through Hirshfeld (overlap) ${ }^{[53]}$ populations (distinguishing covalent and ionic bonds) and the atom size in the mol- 
ecule, and ensures a successful treatment of both weak intra- (short-range) and inter(long range) molecular interactions.

Four test sets assess hydrocarbon thermochemistry: three BSE reaction sets divided into alkane chains, rings and cages (see Fig. 1) and the IDHC (intramolecular dispersion in hydrocarbons ${ }^{[54]}$ ) test set containing the dimerization of anthracene, the hydrogenation reaction of [2.2] paracyclophane to $p$-xylene, two folding reactions of alkane chains $\left(\mathrm{C}_{14} \mathrm{H}_{30}\right.$ and $\left.\mathrm{C}_{22} \mathrm{H}_{46}\right)$ and two isomerization reactions ( $n$-octane and $n$ undecane to their fully branched isomers). In addition, three test sets representative of more typical intra and intermolecular weak interactions were investigated: The S22 test set ${ }^{[55,56]}$ for intermolecular complexes between organic molecules, the EX3 test set for dimers of pnictogen trihalides ${ }^{[18,57]}$ $\left(\mathrm{NF}_{3}, \mathrm{NCl}_{3}, \mathrm{PCl}_{3}, \mathrm{PBr}_{3}, \mathrm{AsBr}_{3}\right)$ and the P76 test set ${ }^{[58]}$ comparing relative conformational stabilities of di- and tripeptides (i.e. FGG, GFA, GGF, WG, WGG). For further details, see ref. [18].

Most computations were performed in a development version of Q-Chem. ${ }^{59]}$ M06-2X ${ }^{[37]}$ and HSE06 ${ }^{[60,61]}$ were run in NWChem 5.1 ${ }^{[62,63]}$ and Gaussian 09, ${ }^{[64]} \mathrm{re}-$ spectively, whereas B97-D ${ }^{[29]}$ and B2PLYP-D ${ }^{[54,65]}$ computations were carried out in Turbomole 5.10.[66,67] LC-BLYP[68] $(\mu=$ 0.33), MCY3 ${ }^{[69]}$ and rCAMB3LYP ${ }^{[69]}$ computations were performed using a modified version of CADPAC ${ }^{[70,71]}$ provided by Dr. Aron Cohen. All the computations use the cc-pVTZ basis. Further details can be found in the original publications. ${ }^{[15,18]}$

\section{Results and Discussion}

As demonstrated by Wodrich and coworkers, alkane reaction energies are poorly described by common density functional approximations. ${ }^{[7,8]}$ Mean absolute deviations (MADs) of standard approximations are shown in Fig. 2 for the hydrocarbon test sets. Whereas all density functionals improve over Hartree-Fock $\left(\mathrm{MAD}=16.9 \mathrm{kcal} \mathrm{mol}^{-1}\right)$, the errors are of the same order of magnitude $(11.0 \mathrm{kcal}$ $\mathrm{mol}^{-1}$ on average). Overall, standard density functional approximations are clearly unable to treat alkane reaction energies adequately. The popular B3LYP ${ }^{[72,73]}$ hybrid functional does not improve upon the simpler PBE[74] GGA functional (MAD = 14.1 and $10.9 \mathrm{kcal} \mathrm{mol}^{-1}$, respectively) and the seemingly good performance the local density approximation (SVWN5 ${ }^{[75,76]}$ ) $\left(\mathrm{MAD}=3.4 \mathrm{kcal} \mathrm{mol}^{-1}\right)$ results form an error cancellation.

The benchmarking of 'modern' functionals is displayed in Fig. 3. The screened hybrid functional HSE06 $($ MAD $=11.0$ kcal mol-1), which is built upon PBE0

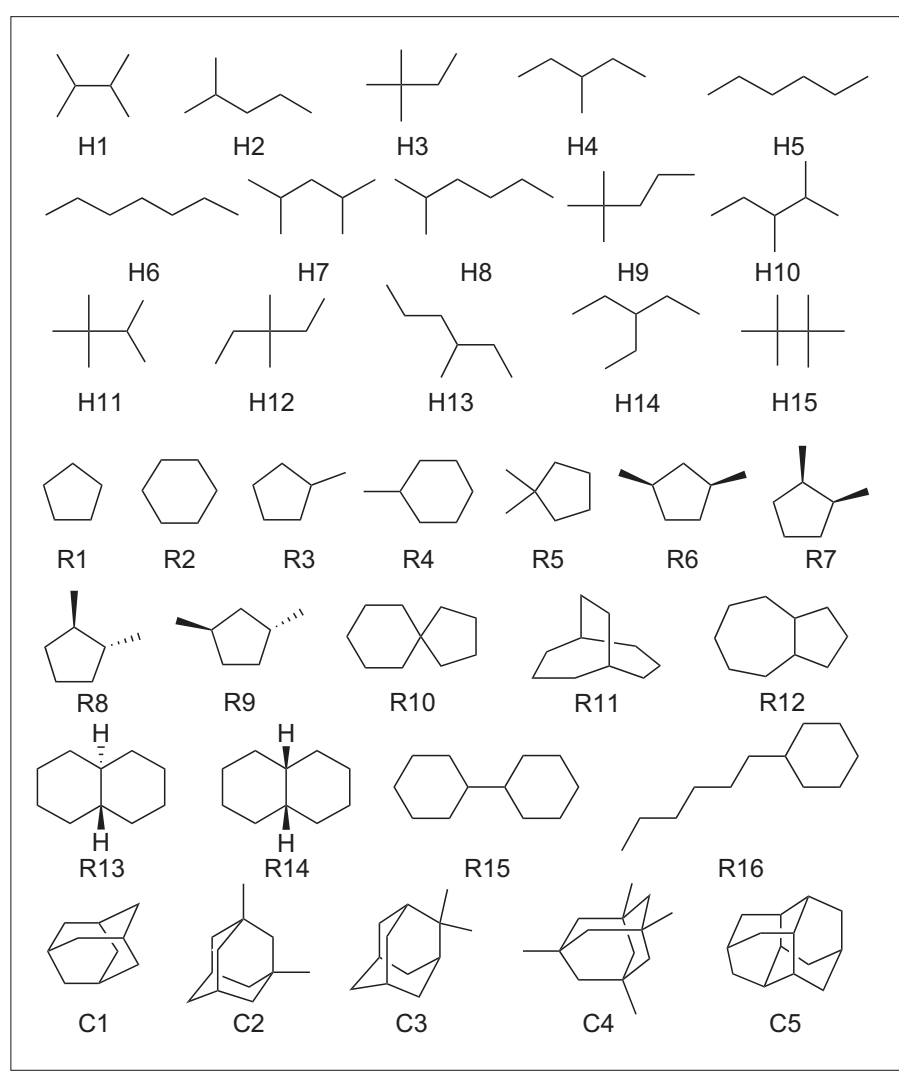

Fig. 1. Schematic representation of the 36 saturated hydrogarbons in the chains $(\mathrm{H} 1-\mathrm{H} 15)$, rings (R1-R16) and cages (C1-C5) test set.

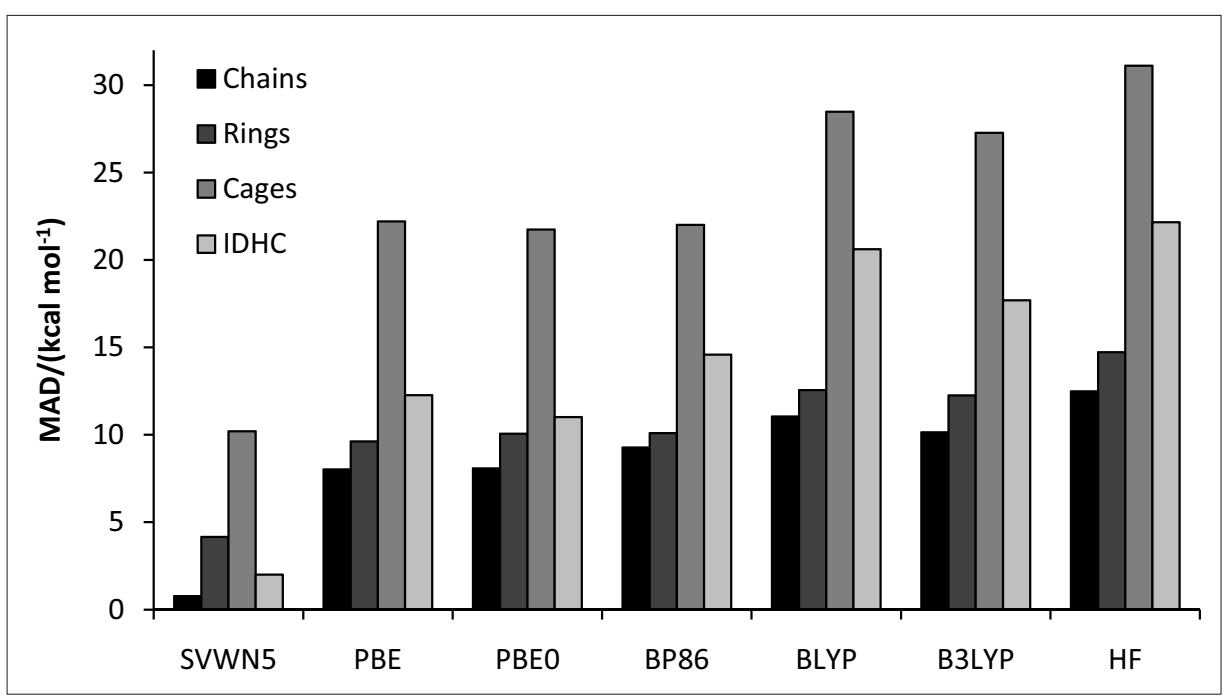

Fig. 2. Performance of standard functionals: Mean absolute deviations for bond separation energies over hydrocarbon chains, rings and cages, and for the reaction energies of the test set 'intramolecular dispersion interaction' (IDHC).

$\left(\mathrm{MAD}=10.9 \mathrm{kcal} \mathrm{mol}^{-1}\right)$, does not outperform its parent functional. In contrast, the long-range corrected exchange variant, $\mathrm{LC}_{-} \omega \mathrm{PBE}^{[77,78]}\left(\mathrm{MAD}=9.0 \mathrm{kcal} \mathrm{mol}^{-1}\right)$ offers an improvement over its standard counterpart (Fig. 3). More generally, the introduction of a long-range correction for the exchange leads to relatively low MADs as illustrated by LC-BLYP, MCY3 and RCAMB3LYP $(5.5,6.3$ and $7.5 \mathrm{kcal}$ $\mathrm{mol}^{-1}$, respectively). We recently demonstrated that the improvements given by the long-range corrected exchange functionals results from a decrease of the overly repulsive nature of the global hy- brid or other standard functionals for describing short-range non-bonded density overlaps ${ }^{[15,79]}$ characteristic of branched alkanes and compact hydrocarbons. The good performance of the meta-GGA functional M06-2X (MAD = $\left.5.6 \mathrm{kcal} \mathrm{mol}^{-1}\right)$, which is probably the most generally successful 'modern' functional, highlights the success of semi-empirical fitting for improving the performance of conventional DFT. ${ }^{[80]}$ Finally, accounting for dispersion interaction by means of a fully non-local density functional (i.e. vdWDF04[40] and VV09[41,81]) lowers the error as well by stabilizing the more compact 
systems. The MADs range from $6.8 \mathrm{kcal}$ $\mathrm{mol}^{-1}$ (revPBE-vdW-DF04) to $3.6 \mathrm{kcal}$ $\mathrm{mol}^{-1}$ (LC-S-VV09).

The atom-pair wise dispersion corrections represent a conceptually and computationally attractive alternative to the fully non-local vdW density functionals. Fig. 4 demonstrates the efficiency of an a posteriori correction for reducing the errors in hydrocarbon reaction energies. Grimme's original system independent dispersion correction $\left(\mathrm{C}_{6}\right.$ only), exemplified by $\mathrm{B} 97-$ $\mathrm{D}\left(\mathrm{MAD}=3.4 \mathrm{kcal} \mathrm{mol}^{-1}\right)$, does improve over standard functionals, but still gives significant errors. The corrected double hybrid B2PLYP-D (i.e. mixing in of perturbation theory correlation energy) reduces the error even more $(\mathrm{MAD}=2.4 \mathrm{kcal}$ $\mathrm{mol}^{-1}$ ), but at higher computational cost. Alternatively, our density dependent dispersion correction dDXDM outperforms all the other variants in correcting a wide variety of standard density functionals giving low errors, e.g. PBE- and B3LYPdDXDM give MADs of 0.8 and $1.3 \mathrm{kcal}$ $\mathrm{mol}^{-1}$, respectively. Interestingly, HFdDXDM (MAD $3.0 \mathrm{kcal} \mathrm{mol}^{-1}$ ) can also compete with M06-2X $(\mathrm{MAD}=5.6 \mathrm{kcal}$ $\mathrm{mol}^{-1}$ ) for these test sets.

More general examples of weak interactions are given by relative conformational energies of di- and tripeptides (P76), [58] the $\mathrm{S} 22$ test set ${ }^{[55]}$ for weakly interacting complexes and five pnictogen trihalide complexes (EX3) (Fig. 5). ${ }^{[57]}$ Established methods for weak interaction energies such as $\mathrm{M} 06-2 \mathrm{X}\left(\mathrm{MAD}=0.4 \mathrm{kcal} \mathrm{mol}^{-1}\right)$ or any variant of dispersion correction, including fully non-local $(\mathrm{MAD}=0.6$ and $0.7 \mathrm{kcal}$ $\mathrm{mol}^{-1}$ for revPBE-vdw-DF04 and rPW86VV09, respectively) or Grimme's classical correction $\left(\mathrm{MAD}=0.6\right.$ and $0.5 \mathrm{kcal} \mathrm{mol}^{-1}$ for B97-D and B2PLYP-D, respectively), gives small errors. Unlike in the latter case, the fitting procedure of $\mathrm{dDXDM}$ does not include weakly interacting intermolecular complexes. However, the robustness of our density-dependent correction is validated by its very good performance on the general test sets (MADs of 0.9 and $0.6 \mathrm{kcal}$ mol $^{-1}$ for PBE- and B3LYP-dDXDM, vs. 1.6 and $1.9 \mathrm{kcal} \mathrm{mol}^{-1}$ for the uncorrected functionals).

A clear advantage of the dDXDM correction is its ability to improve standard density functionals simultaneously for hydrocarbon reaction energies and typical weak interaction energies. In contrast, other dispersion corrections perform satisfyingly for typical non-covalent weak interactions but relatively poorly for the intramolecular problem. The enhanced transferability of $\mathrm{dDXDM}$, as compared to classic dispersion corrections, relies upon the flexible, density-dependent damping function that combines the correct asymptotic behaviour needed for

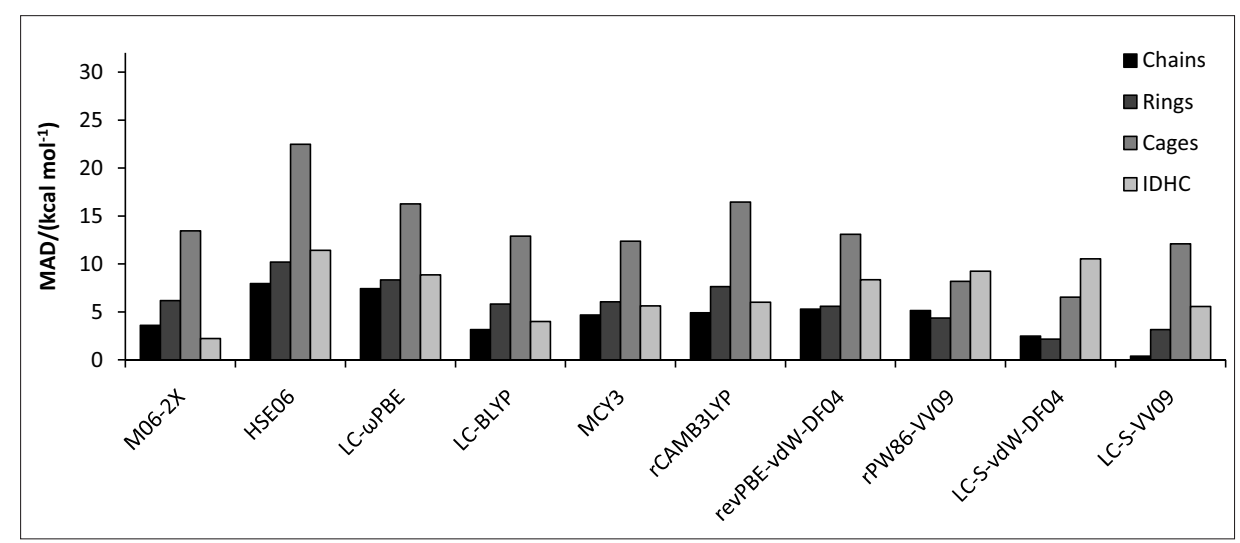

Fig. 3. Performance of 'modern' functionals: Mean absolute deviations for bond separation energies over hydrocarbon chains, rings and cages, and for the reaction energies of the test set 'intramolecular dispersion interaction' (IDHC).

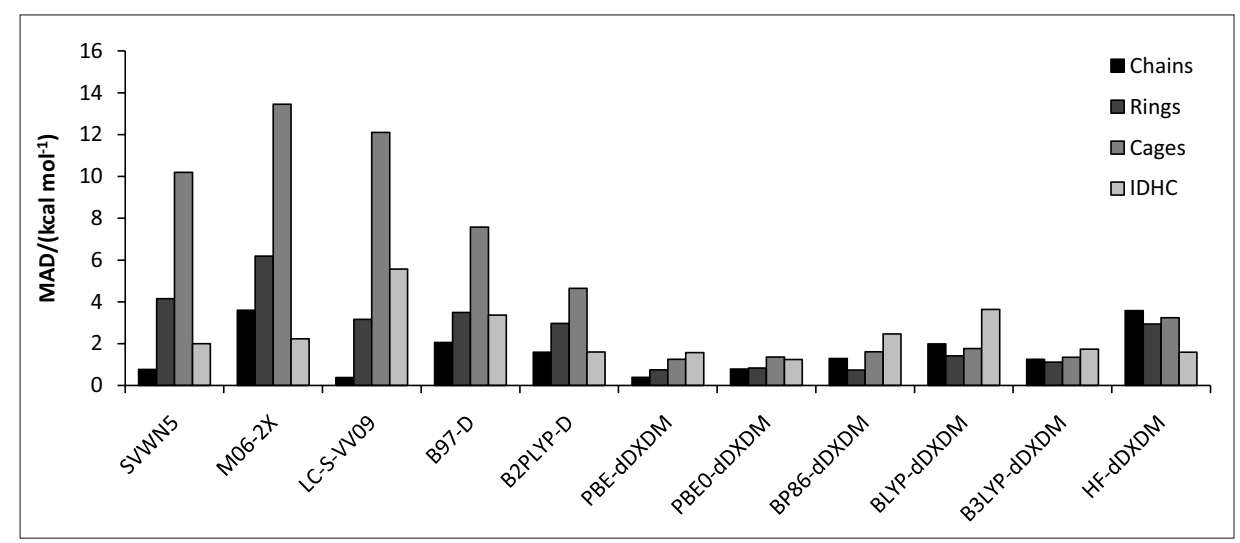

Fig. 4. Performance of dispersion corrected functionals: Mean absolute deviations for bond separation energies over hydrocarbon chains, rings and cages, and for the reaction energies of the test set 'intramolecular dispersion interaction' (IDHC). SVWN5, M06-2X and LC-S-VV09 are shown for comparison with Figs 2 and 3.

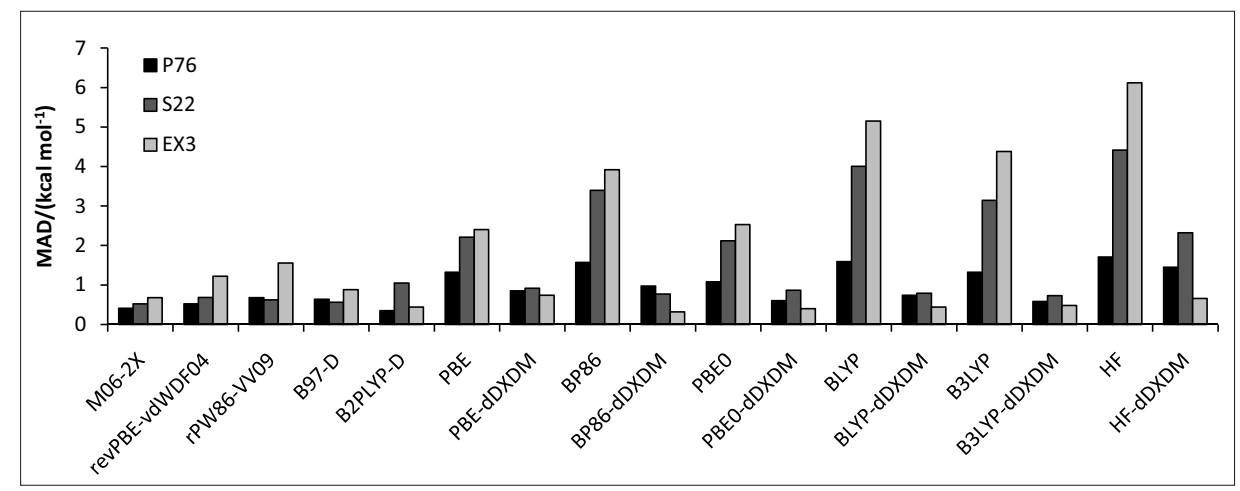

Fig. 5. Performance for weak interaction energies: Mean absolute deviations for reaction energies over relative conformational energies of small di- and tri-peptides (P76), the S22 test set and pnictogen trihalides (EX3).

weakly interacting fragments with a nonnegligible correction at shorter distances. As demonstrated in our former work, $[16,17]$ corrections in the medium range distances are indeed important for intramolecular interactions. Our proposed damping function along with the inclusion of higher-order dispersion coefficients (that contribute significantly to the medium range ${ }^{[17,82,83]}$ ) improve the correction at shorter distances without deteriorating the $\mathrm{C}_{6}$-dominated long-range.

\section{Conclusion}

Alkane reaction energies reveal sizable systematic errors of standard density functionals for intramolecular interactions (e.g. $\left.\operatorname{MAD}(B 3 L Y P)=14.1 \mathrm{kcal} \mathrm{mol}^{-1}\right)$. The substantial improvement offered by modern functionals over standard approximations relies on extensive parameter fitting (e.g. $\left.\operatorname{MAD}(\mathrm{M} 06-2 \mathrm{X})=\mathrm{MAD}=5.6 \mathrm{kcal} \mathrm{mol}^{-1}\right)$, inclusion of a long-range correction (e.g. $\left.\operatorname{MAD}(\mathrm{LC}-\mathrm{BLYP})=5.5 \mathrm{kcal} \mathrm{mol}^{-1}\right)$ or fully 
non-local energy terms (e.g. MAD(rPW86VV09) $\left.=5.8 \mathrm{kcal} \mathrm{mol}^{-1}\right)$. Building on our expertise in intramolecular interactions in alkanes, we devised the robust and computationally inexpensive, dDXDM dispersion correction. The success of $\mathrm{dDXDM}$ relies on i) density dependent dispersion coefficients, which take into account the chemical environment and are available for all elements of the periodic table and ii) the flexible, density-dependent extended Tang and Toennies damping function that allows to simultaneously correct the medium- and the long-range with a minimum of empiricism (two fitted parameters to adapt the correction to a given functional). Standard functionals together with the UDXDM correction (e.g. PBE-dDXDM and B3LYP$\mathrm{dDXDM}$ ) are as accurate for typical weak interactions (e.g. the MAD for the S22 test set is 0.9 and 0.7 , respectively) as for alkane reaction energies $(\mathrm{MAD}=0.8$ and $\left.1.3 \mathrm{kcal} \mathrm{mol}^{-1}\right)$. The dDXDM correction is thus expected to perform well in a broad range of standard applications (e.g. elucidation of reaction mechanisms) and offers possible advantages especially for highlypolarized and charged systems (due to its density dependence).

\section{Acknowledgements}

C. C. and S. N. S. acknowledge the Sandoz family foundation, the Swiss NSF grant 200021 $121577 / 1$ and EPFL for financial support.

Received: January 24, 2011

[1] W. Kohn, L. J. Sham, Phys. Rev. 1965, 140, A1133.

[2] S. Kristyan, P. Pulay, Chem. Phys. Lett. 1994, 229, 175.

[3] J. M. Perez-Jorda, A. D. Becke, Chem. Phys. Lett. 1995, 233, 134.

[4] P. Hobza, J. Sponer, T. Reschel, J. Comput. Chem. 1995, 16, 1315.

[5] Y. Zhang, W. Pan, W. Yang, J. Chem. Phys. 1997, 107, 7921

[6] S. Grimme, Angew. Chem., Int. Ed. 2006, 45, 4460.

[7] M. D. Wodrich, C. Corminboeuf, P. v. R. Schleyer, Org. Lett. 2006, 8, 3631.

[8] M. D. Wodrich, C. Corminboeuf, P. R Schreiner, A. A. Fokin, P. v. R. Schleyer, Org Lett. 2007, 9, 1851.

[9] P. R. Schreiner, Angew. Chem., Int. Ed. 2007, 46, 4217.

[10] Isodesmic bond separation equations are defined by splitting all heavy-atom bonds into their parent molecular fragments preserving the heavy atom bond type. Inclusion of simple hydrides balances the hydrogen-heavy atom bonds (e.g. methane for $\mathrm{C}-\mathrm{H}$ bonds).

[11] J. A. Pople, L. Radom, W. J. Hehre, J. Am. Chem. Soc. 1971, 93, 289.

[12] W. J. Hehre, R. Ditchfield, L. Radom, J. A. Pople, J. Am. Chem. Soc. 1970, 92, 4796.

[13] J.-W. Song, T. Tsuneda, T. Sato, K. Hirao, Org. Lett. 2010, 12, 1440.

[14] S. Grimme, Org. Lett. 2010, 12, 4670.

[15] S. N. Steinmann, M. Wodrich, C. Corminboeuf, Theor. Chem. Acc. 2010, 127, 429.

[16] M. D. Wodrich, D. F. Jana, P. v. R. Schleyer, C. Corminboeuf, J. Phys. Chem. A 2008, 112, 11495.
[17] S. N. Steinmann, G. Csonka, C. Corminboeuf, J. Chem. Theory Comput. 2009, 5, 2950.

[18] S. N. Steinmann, C. Corminboeuf, J. Chem. Theory Comput. 2010, 6, 1990.

[19] J. F. Gonthier, M. D. Wodrich, S. N. Steinmann, C. Corminboeuf, Org. Lett. 2010, 12, 3070.

[20] X. Wu, M. C. Vargas, S. Nayak, V. Lotrich, G. Scoles, J. Chem. Phys. 2001, 115, 8748.

[21] Q. Wu, W. Yang, J. Chem. Phys. 2002, 116, 515.

[22] S. Grimme, J. Comput. Chem. 2004, 25, 1463.

[23] P. Jurecka, J. Cerny, P. Hobza, D. R. Salahub, J. Comput. Chem. 2007, 28, 555.

[24] I.-C. Lin, M. D. Coutinho-Neto, C. Felsenheimer, O. A. v. Lilienfeld, I. Tavernelli, U. Rothlisberger, Phys. Rev. B 2007, 75, 205131.

[25] O. A. von Lilienfeld, I. Tavernelli, U. Rothlisberger, D. Sebastiani, Phys. Rev. B 2005, 71, 195119.

[26] O. A. von Lilienfeld, I. Tavernelli, U. Rothlisberger, D. Sebastiani, Phys. Rev. Lett. 2004, 93 , 153004.

[27] I. D. Mackie, G. A. DiLabio, J. Phys. Chem. A 2008, 112, 10968.

[28] S. O. Nilsson Lill, J. Phys. Chem. A 2009, 113, 10321.

[29] S. Grimme, J. Comput. Chem. 2006, 27, 1787.

[30] A. D. Becke, E. R. Johnson, J. Chem. Phys. 2005, 122, 154104.

[31] A. D. Becke, E. R. Johnson, J. Chem. Phys. 2005, 123, 154101.

[32] A. D. Becke, E. R. Johnson, J. Chem. Phys. 2006, 124, 014104.

[33] A. Tkatchenko, M. Scheffler, Phys. Rev. Lett. 2009, 102, 073005

[34] T. Sato, H. Nakai, J. Chem. Phys. 2009, 131, 224104.

[35] T. Sato, H. Nakai, J. Chem. Phys. 2010, 133, 194101.

[36] X. Xu, W. A. Goddard, Proc. Natl. Acad. Sci. USA 2004, 101, 2673.

[37] Y. Zhao, D. Truhlar, Theor. Chem. Acc. 2008, 120, 215.

[38] Y. Andersson, D. C. Langreth, B. I. Lundqvist, Phys. Rev. Lett. 1996, 76, 102.

[39] J. F. Dobson, B. P. Dinte, Phys. Rev. Lett. 1996, $76,1780$.

[40] M. Dion, H. Rydberg, E. Schröder, D. C. Langreth, B. I. Lundqvist, Phys. Rev. Lett. 2004, 92,246401

[41] O. A. Vydrov, T. Van Voorhis, Phys. Rev. Lett. 2009, 103, 063004

[42] J. P. Perdew, A. Zunger, Phys. Rev. B 1981, 23, 5048.

[43] P. Mori-Sanchez, A. J. Cohen, W. Yang, J. Chem. Phys. 2006, 124, 091102.

[44] A. Ruzsinszky, J. P. Perdew, G. I. Csonka, O. A. Vydrov, G. E. Scuseria, J. Chem. Phys. 2006, $125,194112$.

[45] P. Mori-Sanchez, A. J. Cohen, W. Yang, J. Chem. Phys. 2006, 125, 201102.

[46] A. Savin in 'On degeneracy, near-degeneracy and density functional theory', Vol. 4, Ed. J. M. Seminario, Elsevier, Amsterdam, 1996, pp. 327.

[47] H. Iikura, T. Tsuneda, T. Yanai, K. Hirao, $J$. Chem. Phys. 2001, 115, 3540.

[48] T. Yanai, D. P. Tew, N. C. Handy, Chem. Phys. Lett. 2004, 393, 51.

[49] E. Weintraub, T. M. Henderson, G. E. Scuseria, J. Chem. Theory Comput. 2009, 5, 754.

[50] P. Bultinck, C. V. Alsenoy, P. W. Ayers, R. Carbo-Dorca, J. Chem. Phys. 2007, 126, 144111.

[51] F. L. Hirshfeld, Theor. Chem. Acc. 1977, 44, 129.

[52] K. T. Tang, J. P. Toennies, J. Chem. Phys. 1984, 80,3726

[53] I. Mayer, P. Salvador, Chem. Phys. Lett. 2004, $383,368$.

[54] T. Schwabe, S. Grimme, Phys. Chem. Chem. Phys. 2007, 9, 3397.

[55] P. Jurecka, J. Sponer, J. Cerny, P. Hobza, Phys. Chem. Chem. Phys. 2006, 8, 1985.
[56] T. Takatani, E. G. Hohenstein, M. Malagoli, M. S. Marshall, C. D. Sherrill, J. Chem. Phys. 2010, 132, 144104 .

[57] J. Moilanen, C. Ganesamoorthy, M. S. Balakrishna, H. M. Tuononen, Inorg. Chem. 2009, 48, 6740 .

[58] H. Valdes, K. Pluhackova, M. Pitonak, J. Rezac, P. Hobza, Phys. Chem. Chem. Phys. 2008, 10, 2747.

[59] Y. Shao, L. F. Molnar, Y. Jung, J. Kussmann, C. Ochsenfeld, S. T. Brown, A. T. B. Gilbert, L. V. Slipchenko, S. V. Levchenko, D. P. O'Neill, R. A. DiStasio Jr, R. C. Lochan, T. Wang, G. J. O. Beran, N. A. Besley, J. M. Herbert, C. Y. Lin, T. V. Voorhis, S. H. Chien, A. Sodt, R. P. Steele, V. A. Rassolov, P. E. Maslen, P. P. Korambath, R. D. Adamson, B. Austin, J. Baker, E. F. C. Byrd, H. Dachsel, R. J. Doerksen, A. Dreuw, B. D. Dunietz, A. D. Dutoi, T. R. Furlani, S. R. Gwaltney, A. Heyden, S. Hirata, C.-P. Hsu, G. Kedziora, R. Z. Khalliulin, P. Klunzinger, A. M. Lee, M. S. Lee, W. Liang, I. Lotan, N. Nair, B. Peters, E. I. Proynov, P. A. Pieniazek, Y. M. Rhee, J. Ritchie, E. Rosta, C. D. Sherrill, A. C. Simmonett, J. E. Subotnik, H. L. Woodcock III, W. Zhang, A. T. Bell, A. K. Chakraborty, D. M. Chipman, F. J. Keil, A. Warshel, W. J. Hehre, H. F. Schaefer III, J. Kong, A. I. Krylov, P. M. W. Gill, M. Head-Gordon, Phys. Chem. Chem. Phys. 2006, 8, 3172.

[60] J. Heyd, G. E. Scuseria, M. Ernzerhof, J. Chem. Phys. 2006, 124, 219906.

[61] J. Heyd, G. E. Scuseria, M. Ernzerhof, J. Chem. Phys. 2003, 118, 8207

[62] E. J. Bylaska, W. A. de Jong, N. Govind, K. Kowalski, T. P. Straatsma, M. Valiev, D. Wang, E. Apra, T. L. Windus, J. Hammond, P. Nichols, S. Hirata, M. T. Hackler, Y. Zhao, P.-D. Fan, R. J. Harrison, M. Dupuis, D. M. A. Smith, J. Nieplocha, V. Tipparaju, M. Krishnan, Q. Wu, T. Van Voorhis, A. A. Auer, M. Nooijen, E. Brown, G. Cisneros, G. I. Fann, H. Fruchtl, J. Garza, K. Hirao, R. Kendall, J. A. Nichols, K. Tsemekhman, K. Wolinski, J. Anchell, D. Bernholdt, P. Borowski, T. Clark, D. Clerc, H. Dachsel, M. Deegan, K. Dyall, D. Elwood, E. Glendening, M. Gutowski, A. Hess, J. Jaffe, B. Johnson, J. Ju, R. Kobayashi, R. Kutteh, Z. Lin, R. Littlefield, X. Long, B. Meng, T. Nakajima, S. Niu, L. Pollack, M. Rosing, G. Sandrone, M. Stave, H. Taylor, G. Thomas, J. van Lenthe, A. Wong, Z. Zhang NWChem, 'A Computational Chemistry Package for Parallel Computers', Version 5.1, Richland, Washington 993520999, 2007.

[63] R. A. Kendall, E. Aprà, D. E. Bernholdt, E. J. Bylaska, M. Dupuis, G. I. Fann, R. J. Harrison, J. Ju, J. A. Nichols, J. Nieplocha, T. P. Straatsma, T. L. Windus, A. T. Wong, Comput. Phys. Commun. 2000, 128, 260.

[64] Gaussian 09, Revision A.1, M. J. Frisch, G. W. Trucks, H. B. Schlegel, G. E. Scuseria, M. A. Robb, J. R. Cheeseman, G. Scalmani, V. Barone, B. Mennucci, G. A. Petersson, H. Nakatsuji, M. Caricato, X. Li, H. P. Hratchian, A. F. Izmaylov, J. Bloino, G. Zheng, J. L. Sonnenberg, M. Hada, M. Ehara, K. Toyota, R. Fukuda, J. Hasegawa, M. Ishida, T. Nakajima, Y. Honda, O. Kitao, H Nakai, T. Vreven, J. A. Montgomery, Jr., J. E. Peralta, F. Ogliaro, M. Bearpark, J. J. Heyd, E. Brothers, K. N. Kudin, V. N. Staroverov, R. Kobayashi, J. Normand, K. Raghavachari, A. Rendell, J. C. Burant, S. S. Iyengar, J. Tomasi, M. Cossi, N. Rega, J. M. Millam, M. Klene, J. E. Knox, J. B. Cross, V. Bakken, C. Adamo, J. Jaramillo, R. Gomperts, R. E. Stratmann, O. Yazyev, A. J. Austin, R. Cammi, C. Pomelli, J. W. Ochterski, R. L. Martin, K. Morokuma, V. G. Zakrzewski, G. A. Voth, P. Salvador, J. J. Dannenberg, S. Dapprich, A. D. Daniels, O. Farkas, J. B. Foresman, J. V. Ortiz, J. Cioslowski, D. J. Fox, Gaussian, Inc., Wallingford CT, 2009. 
[65] S. Grimme, J. Chem. Phys. 2006, 124, 034108.

[66] R. Ahlrichs, M. Bär, H.-P. Baron, R. Bauernschmitt, S. Böcker, N. Crawford, P. Deglmann, M. Ehrig, K. Eichkorn, S. Elliott, F. Furche, F. Haase, M. Häser, C. Hättig, A. Hellweg, H. Horn, C. Huber, U. Huniar, M. Kattannek, A. Köhn, C. Kölmel, M. Kollwitz, K. May, P. Nava, C. Ochsenfeld, H. Öhm, H. Patzelt, D. Rappoport, O. Rubner, A. Schäfer, U. Schneider, M. Sierka, O. Treutler, B Unterreiner, M. von Arnim, F. Weigend, P. Weis, H. Weiss, TURBOMOLE V5.10, Karlsruhe, 2008.

[67] R. Ahlrichs, M. Bär, M. Häser, H. Horn, C. Kölmel, Chem. Phys. Lett. 1989, 162, 165.

[68] Y. Tawada, T. Tsuneda, S. Yanagisawa, T. Yanai, K. Hirao, J. Chem. Phys. 2004, 120, 8425.

[69] A. J. Cohen, P. Mori-Sanchez, W. Yang, J. Chem. Phys. 2007, 126, 191109.

[70] CADPAC, The Cambridge Analytic Derivatives Package.

[71] $\mathrm{f}$ functions were omitted in CADPAC computations of the anthracene dimerization reaction and the folding of $\mathrm{C} 22 \mathrm{H} 46$ for technical reasons.
[72] A. D. Becke, J. Chem. Phys. 1993, 98, 5648.

[73] P. J. Stephens, F. J. Devlin, C. F. Chabalowski, M. J. Frisch, J. Phys. Chem. 1994, 98, 11623.

[74] J. P. Perdew, K. Burke, M. Ernzerhof, Phys. Rev. Lett. 1996, 77, 3865.

[75] J. C. Slater, Phys. Rev. 1951, 81, 385.

[76] S. H. Vosko, L. Wilk, M. Nusair, Can. J. Phys. 1980, 58, 1200.

[77] T. M. Henderson, B. G. Janesko, G. E. Scuseria, J. Chem. Phys. 2008, 128, 194105.

[78] M. A. Rohrdanz, K. M. Martins, J. M. Herbert, J. Chem. Phys. 2009, 130, 054112.

[79] E. R. Johnson, P. Mori-Sanchez, A. J. Cohen, W. Yang, J. Chem. Phys. 2008, 129, 204112.

[80] Y. Zhao, D. G. Truhlar, Acc. Chem. Res. 2008, $41,157$.

[81] O. A. Vydrov, T. V. Voorhis, J. Chem. Phys. 2010, 132, 164113.

[82] K. T. Tang, J. P. Toennies, J. Chem. Phys. 2003, $118,4976$.

[83] E. R. Johnson, A. D. Becke, J. Chem. Phys. 2006, 124, 174104. 